\title{
Healthcare use in adults with insomnia: a longitudinal study
}

\author{
Richard Hayward, Kelvin P Jordan and Peter Croft
}

\section{ABSTRACT}

\section{Background}

Insomnia is common and associated with high rates of anxiety and depression.

Aim

To determine the association of insomnia with future primary care for sleep and mood problems, and explore the link with psychological distress.

\section{Design of study}

Questionnaire survey of adults aged $\geq 18$ years, linked to primary care records.

Setting

Five UK general practices.

\section{Method}

Survey responses were linked to recorded consultations and prescribed medication for sleep and mood problems for 12 months following the survey. Baseline self-reported insomnia was defined as the presence of at least one of four sleep problems. Psychological distress was measured using the Hospital Anxiety and Depression Scale.

\section{Results}

A total of 2662 (56\%) responded and 2192 (82\%) consented to record review. Nineteen per cent had a subsequent recorded consultation or prescription for sleep or mood problems ('relevant healthcare use') Self-reported insomnia was related to future relevant healthcare use (odds ratio $=1.77 ; 95 \%$ confidence interval $=1.35$ to 2.33 ). This relationship was stronger in those with psychological distress. Sixty-three per cent of those with baseline insomnia and psychological distress had no relevant healthcare use. 'Trouble falling asleep' was most strongly associated with relevant healthcare use in persons without psychological distress; in persons with psychological distress, it was waking after a usual amount of sleep feeling tired and worn out.

\section{Conclusion}

Specific symptoms of insomnia are associated with consultation for sleep or mood problems, regardless of the presence of anxiety and depression. There is a large group of people who have combined insomnia and anxiety or depression who do not contact primary care about either sleep or mood problems.

\section{Keywords}

anxiety; depression; insomnia; longitudinal study; primary care.

\section{INTRODUCTION}

Insomnia is a common symptom in the general population, with a reported prevalence varying from $8 \%$ to $37 \% .^{1-5}$ This wide variation is related to differing criteria used to define the problem.

Insomniacs are high users of the health service, having more consultations, consuming more drugs, and having more hospital admissions than good sleepers. ${ }^{5,6}$ One reason for this might be the close association of insomnia with psychiatric disorders, especially anxiety and depression, ${ }^{1-3,7,8}$ which are also linked with high healthcare use. Insomnia has been reported to be present in up to $80 \%$ of those with a major depressive disorder, ${ }^{9}$ and may be an early symptom of depression. ${ }^{9-11}$

Despite its high prevalence in the population, little is known about the use of primary care for insomnia with or without accompanying psychological distress. One reason for this might be the uncertain effectiveness of treatment. Benzodiazepines may cause addiction, daytime drowsiness, and memory and psychomotor impairment.,12 The more recently introduced Z drugs (zopiclone, zolpidem, and zaleplon) have little proven advantage in terms of effectiveness or side-effects. ${ }^{13}$ Other pharmaceutical treatments for insomnia such as the tricyclic antidepressants, doxepin and trimipramine, and the pineal gland hormone melatonin have all shown

R Hayward, BMBS, PhD, GP research fellow; KP Jordan, $P h D$, reader in biostatistics; $\boldsymbol{P}$ Croft, $M D$, professor of primary care epidemiology, Arthritis Research Campaign National Primary Care Centre, Keele University, Keele.

Address for correspondence

Richard Hayward, Arthritis Research Campaign National Primary Care Centre, Primary Care Sciences,

Keele University, Keele, ST5 5BG.

E-mail: r.hayward@cphc.keele.ac.uk

Submitted: 13 March 2009; Editor's response: 8 May 2009; final acceptance: 20 October 2009.

(c)British Journal of General Practice 2010; 60: 334-340.

DOI: 10.3399/bjgp10X501822 
some effectiveness in improving sleep quality, as have bright light therapy and cognitive behavioural therapy, but studies are of variable quality. ${ }^{14-18}$

This study aimed to determine the extent to which insomniacs in the general population seek primary care for their sleep problem or for mood disorders, and how this is related to the presence of anxiety or depression. The first objective was to assess the relationship of self-reported insomnia symptoms to future use of primary health care for sleep and mood problems. The second objective was to assess whether any relationship of insomnia with such future healthcare use is influenced by the presence of anxiety or depression.

\section{METHOD}

The study was based on a postal survey of a general practice population aged $\geq 18$ years, using a self-completed questionnaire (as previously reported). ${ }^{1}$ One thousand patients per practice were randomly selected by computer program from the registers of each of five general practices (5000 patients in all) in the UK. Following 115 exclusions (currently in hospital, recently died, left the practice, or excluded by their GP as they suffered from severe mental illness), 4885 questionnaires were sent out in April 2000, with reminders at 2 and 5 weeks. The practices cover a mix of urban and rural areas.

Four self-reported questions related to insomnia were included. These were developed by Jenkins et al, and were: 'Over the last month did you (i) have trouble falling asleep, (ii) wake up several times a night, (iii) have trouble staying asleep, (iv) wake up after your usual amount of sleep feeling tired and worn out'?'. ${ }^{19}$ Possible responses were 'not at all', 'on some nights', and 'on most nights'. The study's main definition of insomnia was based on answering one or more of these questions with 'on most nights'.

Anxiety and depression were assessed using the Hospital Anxiety and Depression Scale (HADS). ${ }^{20}$ The HADS consists of seven questions on anxiety and seven on depression. People were classified as having self-reported anxiety or depression if they scored $\geq 8$ on either the anxiety or depression scale. ${ }^{20}$

Insomnia is strongly related to chronic pain, ${ }^{21}$ which may be a confounder of any relationship between poor sleep and use of primary health care. The participants in the baseline survey were asked to shade in a diagram of a blank body manikin for any aches or pains lasting for 1 day or more in the previous month, with the number of pain areas ranging from 0 to 7 . Questions about occupation were also asked, allowing the social class of the participants to be ascertained and rated from I (professional) through to $\mathrm{V}$ (manual). ${ }^{22}$

\section{How this fits in}

Insomnia is a common symptom in the general population and is related to high

healthcare use and psychological distress. However, little is known about the use of primary care for symptoms of insomnia. This longitudinal study linking primary care records to self-reported survey data shows that there are specific symptoms that are associated with future primary care use for sleep and mood problems. However, there is a substantial proportion of the adult population who have existing insomnia symptoms combined with anxiety or depression but who do not present to primary care with these problems.

Participants were also asked for consent to view their medical records. Consultations with the presenting problems of insomnia, anxiety, or depression and relevant prescription(s) issued over the 12 months following the baseline survey were recorded.

All the practices used Read Codes to record morbidity information from consultations. Read Codes are a commonly used morbidity coding system in the UK. Read Codes for insomnia, sleep disturbance, anxiety, depression, and related terms including stress, neurosis, and neurotic were identified by consensus of two GPs, and are hereafter referred to as insomnia and mood problems. Prescription medication was extracted from the records by British National Formulary (BNF) categories 4.1.1 (hypnotics), 4.1.2 (anxiolytics), and 4.3 antidepressants (including 4.3.1 [tricyclics], 4.3.3 [selective serotonin re-uptake inhibitors], 4.3.4 [others - including mirtazipine]). Categories 4.1.3 (barbiturates) and 4.3.2 (monoamine oxidase inhibitors) had not been prescribed to these patients. All antidepressants may be used in the treatment of insomnia. For this study, all antidepressants are grouped into one category, and each of the BNF groups 4.1 .1 (hypnotics, including the $Z$ drugs and shorter-acting benzodiazepines) and 4.1 .2 (anxiolytics, including diazepam) were considered separately.

\section{Analysis}

'Relevant primary healthcare use' was defined as having a record of either a consultation or a prescribed medication for insomnia or mood problem in the 12 months following the baseline survey. The percentage of baseline responders with such subsequent recorded healthcare use was determined. The association of self-reported insomnia at baseline with healthcare use was then assessed, first unadjusted, and then adjusted for self-reported baseline anxiety and depression, age, sex, number of pain areas, and social class. Selfreported insomnia was based on the main definition 
of reporting any one of the four sleep problems. Analysis was performed using logistic regression, with results presented as odds ratios (OR) and 95\% confidence intervals $(\mathrm{Cl})$.

The effect of self-reported insomnia on type of prescription was determined by restricting the analysis to the subgroup of persons who had relevant primary healthcare use. The associations of prescriptions for antidepressants (BNF groups 4.3.1 to 4.3.4), hypnotics (BNF 4.1.1), and anxiolytics (4.1.2) with self-reported baseline insomnia were determined separately, adjusted for baseline anxiety and depression, age, sex, socioeconomic class, and pain.

The second part of the analysis examined whether the relationship of self-reported insomnia with healthcare use varied between those who did and did not report baseline anxiety or depression. Responders were split into two groups and the above analyses repeated separately for each group: (i) those who reported anxiety or depression at baseline, and (ii) those who reported no anxiety or depression.

Finally, in order to assess the specific sleep problems associated with healthcare use, the influence of each of the four individual sleep problems on future healthcare use was assessed after adjusting for age, sex, social class, and number of areas of pain. Further adjustment was then performed for the other sleep problems.

Analysis was performed using SPSS for Windows (version 15.0).

\section{RESULTS}

\section{Response}

The adjusted response was $56 \%$. It was higher in females $(61 \%$ compared to $51 \%$ in males) and in older age groups $(65 \%$ in those aged $\geq 65$ years compared to $43 \%$ in those aged $18-35$ years). Of the 2662 responders at baseline, 2192 (82\%) gave consent for medical record review.

\section{Table 1. Self-reported insomnia ${ }^{a}$ of responders, $\boldsymbol{n}(\%)$.}

\begin{tabular}{lccc} 
& & \multicolumn{2}{c}{ Reported anxiety or depression } \\
\cline { 3 - 4 } & Total $(n=2192)$ & Yes $(n=942)$ & No $(n=1134)$ \\
\hline Insomnia $^{b}$ & $735(34)$ & $503(59)$ & $214(20)$ \\
\hline Trouble falling asleep $^{267(12)}$ & $214(24)$ & $43(4)$ \\
\hline Wake up several times a night & $561(26)$ & $383(43)$ & $158(14)$ \\
\hline Trouble staying asleep & $367(17)$ & $279(32)$ & $77(7)$ \\
\hline Wake up feeling tired and worn out & $424(19)$ & $326(36)$ & $89(8)$ \\
\hline
\end{tabular}

a Defined as 'most nights'. ${ }^{\circ}$ Reported problem on any one or more of the four sleep items. Sum of numbers in Yes and No columns may not equal number in Total column due to missing data.
The mean age of the baseline population of 2192 was 51.3 years (standard deviation [SD] 17.18, median 51 years, range 18-96 years), and $55 \%$ were female. The baseline prevalence of self-reported insomnia for all responders was $37 \%$, as has been reported previously. ${ }^{1}$ Results for the 2192 consenting to record review were virtually identical; 735 (34\%) reported insomnia (that is, at least one sleep problem) on most nights. The most frequent complaint was waking up several times a night (561, $26 \%) ; 503$ (59\%) of those who reported anxiety or depression reported insomnia, compared to 214 $(20 \%)$ without anxiety or depression $\left(\chi^{2}\right.$ test, $P<0.001$ ) (Table 1).

\section{Relevant healthcare use for insomnia and mood problems}

Overall, 421 (19\%) survey responders either consulted for or received a prescription related to sleep or mood problems in the subsequent 12 months. A sleep or mood problem consultation was recorded for 262 (12\%) of the whole study group, although the proportion with a recorded sleep problem during the follow-up period was low $(<1 \%)$. Three-hundred and forty-nine (16\%) survey responders had a recorded prescription in the relevant drug groups, with $12 \%$ receiving an antidepressant, $4 \%$ a hypnotic, and $3 \%$ an anxiolytic (Table 2).

Of those reporting insomnia at baseline, 136 (19\%) had a subsequent relevant consultation (compared to $8 \%$ of those without self-reported insomnia), and $151(21 \%)$ had a prescription for antidepressants (compared to $7 \%$ of those without self-reported insomnia). Rates of hypnotic and anxiolytic prescriptions were also higher in those with selfreported insomnia (Table 2).

Two-hundred and eighteen (30\%) of all persons with baseline insomnia had either a recorded consultation or prescription for a sleep or mood problem in the year following the survey, compared to $12 \%$ of those not reporting insomnia (unadjusted $\mathrm{OR}=3.09 ; 95 \% \mathrm{Cl}=2.44$ to 3.90 ). After adjustment for anxiety and depression, pain, socioeconomic class, age, and sex, the association of self-reported insomnia with future relevant healthcare use was weaker although still statistically significant (adjusted $\mathrm{OR}=1.77 ; 95 \% \mathrm{Cl}=1.35$ to 2.33 ) (Table 3 ).

Reporting anxiety or depression was strongly associated with future relevant healthcare use (unadjusted $\mathrm{OR}=4.56 ; 95 \% \mathrm{Cl}=3.57$ to 5.82 ). In the adjusted analysis (including adjustment for selfreported insomnia), this association with future healthcare use was stronger (adjusted OR $=3.24$; $95 \% \mathrm{Cl}=2.43$ to 4.30 ) than that seen for selfreported insomnia. 


\section{Interaction of insomnia and psychological distress}

Among persons who did not have anxiety or depression at baseline, there was an elevated but non-significant odds of a relevant consultation or prescription for those with any self-reported insomnia problem (adjusted $\mathrm{OR}=1.44 ; 95 \% \mathrm{Cl}=$ 0.88 to 2.35 ) (Table 3).

The increased use of health care in those with insomnias was greater among persons who reported anxiety or depression at baseline (adjusted OR = $1.97 ; 95 \% \mathrm{Cl}=1.41$ to 2.75$)$. However, 317 (63\%) of those with both insomnia and anxiety or depression at baseline had no relevant primary healthcare use recorded in the follow-up year (Table 3).

\section{Patterns of drug prescribing}

In those who had relevant healthcare use, hypnotic prescribing was more closely associated with baseline insomnia in persons who had no anxiety or depression at baseline (adjusted OR $=8.94,95 \% \mathrm{Cl}$ $=2.38$ to 33.65 ) than in persons who did have anxiety or depression (adjusted OR $=2.24 ; 95 \% \mathrm{Cl}=0.95$ to 5.29). The association between insomnia reporting and anxiolytic prescribing was also greater in the group without baseline anxiety or depression, although it was non-significant in both groups (Table 4).

\section{Individual sleep problems}

In persons with no anxiety or depression, the individual sleep problems most strongly associated with relevant healthcare use were trouble falling asleep (adjusted OR $=3.27 ; 95 \% \mathrm{Cl}=1.54$ to 6.15 ), and trouble staying asleep (adjusted OR $=1.92 ; 95 \%$ $\mathrm{Cl}=1.00$ to 3.67$)$. After further adjustment for the other sleep problems, trouble falling asleep was still strongly associated with future healthcare use (adjusted $\mathrm{OR}=3.18 ; 95 \% \mathrm{Cl}=1.27$ to 8.00 ) (Table 5).

\begin{tabular}{|c|c|c|c|}
\hline & \multirow[b]{2}{*}{ Total $(n=2192)$} & \multicolumn{2}{|c|}{ Self-reported insomnia ${ }^{a}$} \\
\hline & & Yes $(n=735)$ & No $(n=1239)$ \\
\hline \multicolumn{4}{|l|}{ Consultations } \\
\hline Mood problem or insomnia & $262(12)$ & $136(19)$ & $98(8)$ \\
\hline \multicolumn{4}{|l|}{ Prescriptions } \\
\hline \multicolumn{4}{|l|}{ Antidepressants } \\
\hline 4.3.1 Tricyclics & $109(5)$ & $65(9)$ & $29(2)$ \\
\hline 4.3.3 SSRls & $174(8)$ & $99(13)$ & $55(4)$ \\
\hline 4.3.4 Other antidepressants & 25 (1) & $16(2)$ & $7(<1)$ \\
\hline Any antidepressants & $270(12)$ & $151(21)$ & $85(7)$ \\
\hline \multicolumn{4}{|l|}{ Hypnotics/anxiolytics } \\
\hline 4.1.1 Hypnotics & $87(4)$ & $58(8)$ & $15(1)$ \\
\hline 4.1.2 Anxiolytics & $66(3)$ & $32(4)$ & $23(2)$ \\
\hline Any of the above prescriptions & $349(16)$ & $190(26)$ & $113(9)$ \\
\hline $\begin{array}{l}\text { Any of the above consultations } \\
\text { or prescriptions }\end{array}$ & $421(19)$ & $218(30)$ & $149(12)$ \\
\hline
\end{tabular}

There were no recorded prescriptions for BNF 4.3.2 monoamine oxidase inhibitors or 4.1.3 barbiturates. ${ }^{a}$ Reported problem on any one or more of the four sleep items. Sum of numbers in Yes and No columns may not equal number in Total column due to missing data. SSRI = selective serotonin reuptake inhibitor.

In persons with baseline anxiety and depression, the symptom most strongly associated with future healthcare use was waking up after the usual amount of sleep feeling tired and worn out (adjusted $\mathrm{OR}=1.93 ; 95 \% \mathrm{Cl}=1.41$ to 2.63 ), but having trouble falling asleep and waking up several times were also significantly associated. After further adjustment for the other sleep problems, reporting waking up feeling tired and worn out and reporting waking several times were both independently associated with future healthcare use (Table 5).

In the anxious or depressed group who did seek relevant health care, trouble falling asleep was strongly associated with a hypnotic prescription (adjusted $\mathrm{OR}=3.78 ; 95 \% \mathrm{Cl}=1.92$ to 7.43 ). There was greater use of antidepressants in the group

\section{Table 3. Association between reporting insomnia symptom and a recorded mood or insomnia consultation, or prescription in the following 12 months.}

\begin{tabular}{|c|c|c|c|c|}
\hline Self-report of insomnia & $n$ & $\begin{array}{c}\text { Consultation or } \\
\text { prescription, }{ }^{\text {b }} n(\%)\end{array}$ & $\mathrm{OR}^{\circ}(95 \% \mathrm{Cl})$ & OR $(95 \% \mathrm{Cl})$ \\
\hline \multicolumn{5}{|l|}{ All responders } \\
\hline No & 1239 & $149(12)$ & 1.00 & $1.00^{\mathrm{d}}$ \\
\hline Yes & 735 & $218(30)$ & 3.09 (2.44 to 3.90$)$ & 1.77 (1.35 to 2.33$)$ \\
\hline \multicolumn{5}{|c|}{ Self-report no anxiety or depression } \\
\hline No & 853 & $69(8)$ & 1.00 & $1.00^{\circ}$ \\
\hline Yes & 214 & $27(13)$ & 1.64 (1.02 to 2.63$)$ & 1.44 (0.88 to 2.35$)$ \\
\hline \multicolumn{5}{|c|}{ Self-report anxiety or depression } \\
\hline No & 349 & 77 (22) & 1.00 & $1.00^{\mathrm{e}}$ \\
\hline Yes & 503 & $186(37)$ & 2.07 (1.52 to 2.83$)$ & 1.97 (1.41 to 2.75$)$ \\
\hline
\end{tabular}

${ }^{a}$ Reported problem on any one or more of the four sleep items on 'most nights'. 'Defined as anxiety, depression, or insomnia consultation or prescription for antidepressant, hypnotic or anxiolytic. 'Unadjusted. ${ }^{\circ}$ Adjusted for anxiety or depression, age, sex, socioeconomic class, and number of areas of pain. ${ }^{\circledR}$ Adjusted for age, sex, socioeconomic class, and number of areas of pain. 


\begin{tabular}{|c|c|c|c|c|c|c|c|}
\hline \multirow[b]{2}{*}{ Self-reported insomnia } & \multirow[b]{2}{*}{ Total } & \multicolumn{2}{|c|}{ Antidepressant, } & \multicolumn{2}{|c|}{ Hypnotic 4.1.1, } & \multicolumn{2}{|c|}{ Anxiolytic 4.1.2, } \\
\hline & & $n(\%)$ & OR $(95 \% \mathrm{Cl})$ & $n(\%)$ & OR $(95 \% \mathrm{Cl})$ & $n(\%)$ & OR $(95 \% \mathrm{Cl})$ \\
\hline \multicolumn{8}{|l|}{ All responders } \\
\hline No & 149 & $85(57)$ & $1.00^{\mathrm{b}}$ & $15(10)$ & $1.00^{\mathrm{b}}$ & $23(15)$ & $1.00^{\mathrm{b}}$ \\
\hline Yes & 218 & $151(69)$ & 1.43 (0.86 to 2.39$)$ & $58(27)$ & 3.73 (1.76 to 7.88$)$ & $32(15)$ & 1.33 (0.67 to 2.67 ) \\
\hline \multicolumn{8}{|c|}{ Self-report no anxiety or depression } \\
\hline No & 69 & $36(52)$ & $1.00^{\circ}$ & $5(7)$ & $1.00^{\circ}$ & $13(19)$ & $1.00^{\circ}$ \\
\hline Yes & 27 & $16(59)$ & $1.23(0.48$ to 3.14$)$ & $10(37)$ & 8.94 (2.38 to 33.65$)$ & $8(30)$ & 2.36 (0.78 to 7.14$)$ \\
\hline \multicolumn{8}{|c|}{ Self-report anxiety or depression } \\
\hline No & 77 & $48(62)$ & $1.00^{\circ}$ & $10(13)$ & $1.00^{\circ}$ & $9(12)$ & $1.00^{\circ}$ \\
\hline Yes & 186 & $131(70)$ & 1.51 (0.82 to 2.78$)$ & $46(25)$ & $2.24(0.95$ to 5.29$)$ & $24(13)$ & 0.88 (0.36 to 2.11$)$ \\
\hline
\end{tabular}

${ }^{a}$ Reported problem on any one or more of the four sleep items on 'most nights'. ${ }^{b}$ Adjusted for self-reported anxiety and depression, age, sex, socioeconomic class, and number of areas of pain. 'Adjusted for age, sex, socioeconomic class, and number of areas of pain.

complaining of waking up feeling tired and worn out, but this was not statistically significant (adjusted OR $=1.49 ; 95 \% \mathrm{Cl}=0.84$ to 2.63 ).

\section{DISCUSSION}

\section{Summary of main findings}

This study investigated the association of selfreported sleep problems with primary care consultations and prescriptions related to insomnia and mood problems in the following 12 months.
Almost one-third of those reporting insomnia at baseline presented in primary care over the next 12 months with an insomnia or mood-related problem. However, the symptoms associated with consultation varied depending on the existence of anxiety or depression. In those without anxiety or depression, trouble falling asleep was the main factor associated with consultation; in those reporting anxiety or depression, the main factor was waking up feeling tired and worn out. Prescription of hypnotics

\begin{tabular}{|c|c|c|c|c|c|}
\hline Reported symptoms & $n$ & Consultation or prescription, ${ }^{\mathrm{b}} n(\%)$ & $\mathrm{OR}^{\mathrm{c}}(95 \% \mathrm{Cl})$ & $\mathrm{OR}^{\mathrm{d}}(95 \% \mathrm{Cl})$ & $\mathrm{OR}^{\mathrm{e}}(95 \% \mathrm{Cl})$ \\
\hline \multicolumn{6}{|c|}{ Self-report no anxiety or depression } \\
\hline \multicolumn{6}{|l|}{ Trouble falling asleep } \\
\hline No & 1073 & $90(8)$ & 1.00 & 1.00 & 1.00 \\
\hline Yes & 43 & $10(23)$ & 3.31 (1.58 to 6.94$)$ & 3.27 (1.54 to 6.15$)$ & 3.18 (1.27 to 8.00$)$ \\
\hline \multicolumn{6}{|c|}{ Wakes up several times } \\
\hline No & 944 & $78(8)$ & 1.00 & 1.00 & 1.00 \\
\hline Yes & 158 & $21(13)$ & 1.70 (1.02 to 2.85$)$ & 1.46 (0.85 to 2.51$)$ & 1.10 (0.52 to 2.32 ) \\
\hline \multicolumn{6}{|l|}{ Trouble staying asleep } \\
\hline No & 1011 & $87(9)$ & 1.00 & 1.00 & 1.00 \\
\hline Yes & 77 & $13(17)$ & 2.16 (1.14 to 4.07$)$ & $1.92(1.00$ to 3.67$)$ & 1.06 (0.38 to 2.90$)$ \\
\hline \multicolumn{6}{|c|}{ Wakes feeling tired and worn out } \\
\hline No & 1020 & $92(9)$ & 1.00 & 1.00 & 1.00 \\
\hline Yes & 89 & $10(11)$ & 1.28 (0.64 to 2.55$)$ & 1.19 (0.58 to 2.45$)$ & 0.97 (0.45 to 2.13$)$ \\
\hline \multicolumn{6}{|c|}{ Self-report anxiety or depression } \\
\hline \multicolumn{6}{|l|}{ Trouble falling asleep } \\
\hline No & 683 & $189(28)$ & 1.00 & 1.00 & 1.00 \\
\hline Yes & 214 & $86(40)$ & 1.76 (1.27 to 2.42$)$ & 1.56 (1.10 to 2.21$)$ & 1.30 (0.85 to 2.00$)$ \\
\hline \multicolumn{6}{|c|}{ Wakes up several times } \\
\hline No & 513 & $134(26)$ & 1.00 & 1.00 & 1.00 \\
\hline Yes & 383 & $142(37)$ & 1.67 (1.25 to 2.22$)$ & 1.58 (1.16 to 2.14$)$ & 1.61 (1.06 to 2.45$)$ \\
\hline \multicolumn{6}{|l|}{ Trouble staying asleep } \\
\hline No & 590 & $173(29)$ & 1.00 & 1.00 & 1.00 \\
\hline Yes & 279 & $98(35)$ & $1.31(0.96$ to 1.77$)$ & 1.24 (0.90 to 1.70$)$ & 0.58 (0.36 to 0.95$)$ \\
\hline \multicolumn{6}{|c|}{ Wakes tired and worn out } \\
\hline No & 577 & $148(26)$ & 1.00 & 1.00 & 1.00 \\
\hline Yes & 326 & $132(41)$ & 1.97 (1.48 to 7.64$)$ & 1.93 (1.41 to 2.63$)$ & 1.84 (1.30 to 2.62$)$ \\
\hline
\end{tabular}

a'Defined as 'most nights'. 'Defined as anxiety, depression, or insomnia consultation or prescription for antidepressant, hypnotic, or anxiolytic. 'Unadjusted. 'Adjusted for age, sex, socioeconomic class, and number of areas of pain. ${ }^{\circ}$ Adjusted for age, sex, socioeconomic class, number of areas of pain, and other insomnia symptoms. 
in those with insomnia was greater in those without concordant anxiety or depression.

\section{Strengths and limitations of the study}

This is a novel longitudinal study using self-reported survey data linked with medical record data. Previous studies on sleep disorders have tended to be cross-sectional, and the need for longitudinal studies has been stated. ${ }^{9}$ Associations were adjusted for age, sex, number of pain areas, and social class, all of which have been shown to be related to both insomnia and healthcare use. ${ }^{6}$

This was a secondary analysis of a database constructed in $\mathbf{2 0 0 0}$ for another purpose, the natural history of headache, but with a general health content. The response was $56 \%$, of which $82 \%$ consented to record review, and there were some age and sex differences in response. There may be concern that the rate of GP consultations observed after a postal survey is influenced by the survey itself However, the focus of the survey on headache should not have made those with sleeping problems more likely to respond or have prompted people to consult for insomnia, or anxiety and depression. Previously it has been shown that postal surveys have no effect on subsequent consultation rates, either for the problem under focus or for other problems. ${ }^{23}$ Further, people consenting to record review in this study had similar consultation rates to their whole-practice population. ${ }^{24}$ Standardising the prevalence of insomnia for age and sex did not change observed rates, ${ }^{1}$ and, while any selective non-response may affect the overall estimates of rates of consultation and prescription, the study's central analysis (the strength of association between baseline survey characteristics and subsequent consultation) is unlikely to be affected by any selection differences between participants, nonresponders, and non-consenters. Consultation data were only identified for a period of 12 months after the survey, so it was not possible to ascertain whether healthcare use was new or ongoing; further, it may be that sleep problems and anxiety and depression are less likely to be recorded in multiple problem contacts. The study was set in one area of the UK, which is more deprived than the UK as a whole. However, the use of five general practices should reduce the likelihood that observed healthcare use was influenced by unusual practice characteristics.

\section{Comparison with existing literature}

The prevalence of insomnia in this study was similar to that reported elsewhere using similar questions. ${ }^{2,9}$ One in five insomniacs had a prescribed antidepressant in the course of the next 12 months, similar to the proportion reported by Simon and Von Korff, who described $19 \%$ antidepressant use in current insomniacs. ${ }^{25}$

In the group with anxiety or depression at baseline, the single sleep question most predictive of future healthcare use was waking after a usual amount of sleep feeling tired and worn out. This describes persons with daytime sequelae of insomnia. This relationship was weaker in those not reporting anxiety or depression. These results fit with this particular sleep symptom being a specific feature of anxiety and depression, as reported in clinical texts and in the findings of Jenkins et al, who found that 'wakes up tired' was positively related to selfreported depression, ${ }^{19}$ and Taylor et al, who reported that depression was associated with an increased number of awakenings. ${ }^{8} \mathrm{~A}$ polysomnographic study found that it was sleep fragmentation that produced the daytime symptoms of fatigue. ${ }^{26}$

By contrast, in persons without anxiety or depression at baseline, the main link with relevant healthcare use was having trouble falling asleep. This coincides with the finding that members of this group were far more likely to receive a hypnotic. This use of hypnotics accords with current practice, to avoid daytime symptoms of somnolence. ${ }^{27}$

\section{Implications for clinical practice and future research}

A large group of people had anxiety or depression at baseline and also complained of insomnia but did not have a consultation or prescription in the following year ( $63 \%$ in this population sample). This may include people who recover or have less severe symptoms, but is likely to also include people with possible unmet need with respect to sleep and depression, who may benefit from medical intervention such as an antidepressant or cognitive behavioural therapy. In particular, they may be waking up still feeling tired, with subsequent effects on their social and work activities. It may be that individuals in this group have previously sought health care for this problem, and future research might usefully be directed to assess prognosis in this group.

Insomnia increases healthcare use for sleep or mood problems in persons with and without accompanying anxiety or depression, but there remains a substantial proportion of the adult population ( $14 \%$ in this study's estimation) who have existing insomnia symptoms combined with anxiety or depression, but who do not present to primary care with these problems and hence may have unmet need. It appears that certain features of insomnia are more strongly associated with consultation than others, which needs to be 
recognised in primary care management. The relationship of insomnia to anxiety and depression, and to other symptoms such as chronic pain, suggests sleep management in primary care should be more targeted. Future research might usefully investigate the effectiveness of primary care treatments of insomnia.

\section{Funding body}

The Proprietary Association of Great Britain funded the baseline survey. Other study costs were funded by the arc National Primary Care Centre and the Department of Medicines Management, Keele University, and the North Staffordshire Primary Care Consortium. Richard Hayward is receiving an in-practice fellowship from the National Coordinating Centre for Research Capacity Development (NCCRCD).

\section{Ethical approval}

North Staffordshire Local Research Committee.

\section{Competing interests}

The authors have stated that there are none.

\section{Acknowledgements}

Helen Boardman who conducted the original survey, and patients and staff at the practices involved.

\section{Discuss this article}

Contribute and read comments about this article on the Discussion Forum: http://www.rcgp.org.uk/bjgp-discuss

\section{REFERENCES}

1. Morphy H, Dunn KM, Lewis M, et al. Epidemiology of insomnia: a longitudinal study in a UK population. Sleep 2007; 30(3): 274-280.

2. Stewart R, Besset A, Bebbington P, et al. Insomnia comorbidity and impact and hypnotic use by age group in a national survey population aged 16 to 74 years. Sleep 2006; 29(11): 1391-1397.

3. Janson C, Lindberg E, Gislason T, et al. Insomnia in men - a 10-year prospective population based study. Sleep 2001; 24(4): 425-430.

4. Pallesen S, Nordhus IH, Nielsen GH, et al. Prevalence of insomnia in the adult Norwegian population. Sleep 2001; 24(7): 771-779.

5. Leger D, Guilleminault C, Bader G, et al. Medical and socioprofessional impact of insomnia. Sleep 2002; 25(6): 625-629.

6. Chevalier H, Los F, Boichut D, et al. Evaluation of severe insomnia in the general population: results of a European multinational survey. $J$ Psychopharmacol 1999; 13(4 suppl 1): S21-S24.

7. Benca RM. Diagnosis and treatment of chronic insomnia: a review. Psychiatr Serv 2005; 56(3): 332-343.
8. Taylor DJ, Lichstein KL, Durrence HH, et al. Epidemiology of insomnia, depression, and anxiety. Sleep 2005; 28(11): 1457-1464.

9. Ohayon MM. Epidemiology of insomnia: what we know and what we still need to learn. Sleep Med Rev 2002; 6(2): 97-111.

10. Foley DJ, Monjan A, Simonsick EM, et al. Incidence and remission of insomnia among elderly adults: an epidemiologic study of 6800 persons over three years. Sleep 1999; 22(suppl 2): S366-S372.

11. Lustberg L, Reynolds CF. Depression and insomnia: questions of cause and effect. Sleep Med Rev 2000; 4(3): 253-262.

12. Neutel CI, Perry S, Maxwell C. Medication use and risk of falls. Pharmacoepidemiol Drug Saf 2002; 11(2): 97-104.

13. Glass J, Lanctot KL, Herrmann N, et al. Sedative hypnotics in older people with insomnia: meta-analysis of risks and benefits. BMJ 2005 331(7526): 1169.

14. Hajak G, Rodenbeck A, Voderholzer U, et al. Doxepin in the treatment of primary insomnia: a placebo-controlled, double-blind, polysomnographic study. J Clin Psychiatry 2001; 62(6): 453-463.

15. Riemann D, Voderholzer U, Cohrs S, et al. Trimipramine in primary insomnia: results of a polysomnographic double-blind controlled study. Pharmacopsychiatry 2002; 35(5): 165-174.

16. Brzezinski A, Vangel MG, Wurtman RJ, et al. Effects of exogenous melatonin on sleep: a meta-analysis. Sleep Med Rev 2005; 9(1): 41-50.

17. Montgomery P, Dennis J. Bright light therapy for sleep problems in adults aged 60+. Cochrane Database Syst Rev 2002; (2): CD003403.

18. Smith MT, Perlis ML, Park A, et al. Comparative meta-analysis of pharmacotherapy and behavior therapy for persistent insomnia. Am J Psychiatry 2002; 159(1): 5-11.

19. Jenkins CD, Stanton BA, Niemcryk SJ, Rose RM. A scale for the estimation of sleep problems in clinical research. J Clin Epidemiol 1988; 41(4): 313-321.

20. Zigmond AS, Snaith RP. The hospital anxiety and depression scale. Acta Psychiatr Scand 1983; 67(6): 361-370.

21. Ohayon M. Relationship between chronic painful physical conditions and insomnia. J Psychiatr Res 2005; 39(2): 151-159.

22. Standard occupational classification. Social classifications and coding methodology. London: HMSO, 1991.

23. Jeffery A, Jinks $C$, Jordan $\mathrm{K}$. The influence of completing a healthrelated questionnaire on primary care consultation behaviour. $B M C$ Health Serv Res 2006; 6: 101.

24. Boardman HF, Thomas E, Ogden H, et al. A method to determine if consenters to population surveys are representative of the target study population. J Public Health 2005; 27(2): 212-214.

25. Simon GE, VonKorff M. Prevalence, burden, and treatment of insomnia in primary care. Am J Psychiatry 1997; 154(10): 1417-1423.

26. Stepanski E, Lamphere J, Badia P, et al. Sleep fragmentation and daytime sleepiness. Sleep 1984; 7(1): 18-26.

27. National Institute for Health and Clinical Excellence (NICE). Guidance on the use of Zaleplon, Zolpidem and Zopiclonefor the short term management of insomnia. TA77, 2.3. London: NICE, 2004. 